\section{Delaying radical cystectomy by more than 12 weeks increases risk of mortality}

Delays between diagnosis of stage II muscleinvasive bladder cancer and radical cystectomy can negatively affect the chance of survival according to some reports. Gore et al. sought to confirm these findings using nationally representative data from the linked Surveillance Epidemiology and End Results-Medicare dataset.

Data from 441 patients with stage II transitional cell carcinoma of the bladder who underwent radical cystectomy between 1992 and 2001 were assessed. Surgery was carried out within 24 weeks of diagnosis in $89 \%$ of patients, of whom $73 \%$ underwent surgery within 12 weeks. During the study period, 279 deaths were reported, 136 of which were due to bladder cancer.

The authors compared patient outcomes according to delays of 8,12 , and 24 weeks. Compared with patients who underwent cystectomy within 4-8 weeks, those who waited longer than 12 weeks were at an increased risk of both disease-specific and overall mortality. The risk of mortality for patients delayed for longer than 12 weeks was around twice that of those who waited less than 8 weeks for the procedure.

Gore and colleagues explained that delays between diagnosis and surgical resection of muscle-invasive bladder cancers might relate to the requirement for extensive medical evaluation and clearance or transferal to specialist centers that is often needed before cystectomy can be carried out.

Original article Gore JL et al. (2009) Mortality increases when radical cystectomy is delayed more than 12 weeks: results from a Surveillance, Epidemiology, and End ResultsMedicare analysis. Cancer [doi:101002.cncr.24052]

\section{Vitamin C, vitamin E and selenium supplementation does not prevent prostate cancer}

The results of two large clinical trials, which assessed the effects of antioxidant supplementation on cancer in men, have found no protective effect from vitamins $C$ and $E$ and selenium. These results are contrary to previous studies, which indicated that antioxidant supplements might reduce cancer risk or mortality.

In the Selenium and Vitamin E Cancer Prevention Trial (SELECT), consisting of 35,533 men from the US, Canada and Puerto Rico, Lippman and colleagues investigated the effects of supplementation with vitamin $\mathrm{E}$ (400 IU per day all rac- $\alpha$-tocopheryl acetate) and/or selenium $(200 \mu \mathrm{g}$ per day $L$-selenomethionine). A data review after a median follow-up of 5.46 years revealed that vitamin $\mathrm{E}$ or selenium, taken individually or in combination, did not prevent prostate cancer.

Gaziano et al. reported the outcomes of the Physicians' Health Study II, which assessed the effects of vitamin C $(500 \mu \mathrm{g}$ per day synthetic ascorbic acid) and vitamin E (400IU synthetic a-tocopheryl every second day) supplementation in 14,641 male physicians. After a mean follow-up of 8 years, neither vitamin was seen to affect the risk or outcomes of any cancer, including prostate cancer.

The authors of both trials suggest that antioxidant supplementation at the tested doses is not recommended for cancer prevention, and encourage further research to identify alternative agents that might help to prevent prostate cancer and other cancer in men.

Original articles Lippman SM et al. (2009) Effect of selenium and vitamin $\mathrm{E}$ on risk of prostate cancer and other cancers: the Selenium and Vitamin E Cancer Prevention Trial (SELECT). JAMA 301: 39-51

Gaziano JM et al. (2009) Vitamins E and C in the prevention of prostate and total cancer in men: the Physicians' Health Study II randomized controlled trial. JAMA 301: 52-62

\section{Alfuzosin does not alleviate symptoms of prostatitis-chronic pelvic pain syndrome}

Alpha-blockers ( $\alpha$-adrenergic receptor antagonists) are often prescribed to men with chronic prostatitis-chronic pelvic pain syndrome, despite inconclusive evidence from clinical trials supporting the efficacy of such agents in this patient population. Nickel and colleagues have now assessed whether treatment with the alpha-blocker alfuzosin could alleviate symptoms of chronic prostatitis-chronic pelvic pain syndrome in men.

The study recruited a total of 272 men (mean age 40.1 years) with recently diagnosed (within 2 years before enrollment) chronic 